\title{
Tumor necrosis factor alpha - an useful biomarker in a combined predictive model for liver fibrosis staging in patients with chronic HCV infection
}

\author{
Daniela-loana Munteanu ${ }^{1 *}$, Raluca Mihăilescu', Mihaela Andreea Rădulescu², Anca-Ruxandra Negru', \\ Cătălin Tilişcan ${ }^{1,2}$, Viorica Poghirc ${ }^{1}$, Victoria Aramă ${ }^{1,2}$, Ștefan Sorin Aramă2 \\ From The 10th Edition of the Scientific Days of the National Institute for Infectious Diseases "Prof Dr Matei \\ Bals" \\ Bucharest, Romania. 15-17 October 2014
}

\section{Background}

Staging liver fibrosis in chronic HCV infection represents an important step for an individualized management. In the last decade the liver biopsy was less used for fibrosis staging due to its invasive nature and risk of complications. Multiple non-invasive methods were developed for the evaluation of liver fibrosis, none of these being an ideal one. The aim of this study was to evaluate the diagnostic accuracy of a new non-invasive method designed to differentiate patients with significant liver fibrosis from those without. (F2-F4 vs. F0-F1).

\section{Methods}

We conducted a cross-sectional study in patients with chronic HCV infection, in a tertiary-care hospital, from November 2012 until April 2013. Blood samples were collected for: aspartat-aminotransferase (AST), alaninaminotransferase (ALT), gamma-glutamyl transpeptidase (GGT), total bilirubin (TB), albumin, total cholesterol $(\mathrm{CH})$, triglycerides (TG), fasting glucose (GLU), white blood cells (WBC), platelets (PLT) and tumor necrosis factor alpha (TNFa). We used DIAsource ImmunoAssay, Louvain-la-Neuve, Belgium, for TNFa plasma levels quantification. Liver fibrosis was estimated in all patients using Fibromax ${ }^{\circledR}$ (Biopredictive, France).

\footnotetext{
* Correspondence: danielaioana.mn@gmail.com

"National Institute for Infectious Diseases "Prof. Dr. Matei Balş", Bucharest, Romania

Full list of author information is available at the end of the article
}

\section{Results}

We included 114 consecutive patients and we divided them into 2 groups: estimation group - 79 patients and validation group -35 patients. There were no significant differences between the 2 groups regarding sex ratio, median age, liver fibrosis score or biochemical and inflammation variables. We found a statistical correlation between the liver fibrosis score estimated by Fibromax and age, AST, ALT, GGT, TB, CH, GLU, PLT and TNFa. We constructed a regression model and subsequently a score combining age, TB, PLT count and TNFa that proved to be useful for identification of patients with significant liver fibrosis. The area under the ROC curve was 0.887 for the estimation group and 0.875 for the validation group. Using the best cut-off value $(<1.06)$ the score positive predictive value was $90 \%$. In the estimation group 51\% (40/79) of patients were diagnosed with significant fibrosis whereas in the validation group the percent of patients with significant fibrosis was 54\% (19/35).

\section{Conclusion}

A score combining age of the patient, TB, PLT count and TNFa value could be an accessible and accurate tool for the identification of significant liver fibrosis in patients with chronic HCV infection.

\footnotetext{
Authors' details

'National Institute for Infectious Diseases "Prof. Dr. Matei Balş", Bucharest, Romania. ${ }^{2}$ Carol Davila University of Medicine and Pharmacy, Bucharest, Romania.
} 
doi:10.1186/1471-2334-14-S7-016

Cite this article as: Munteanu et al.: Tumor necrosis factor alpha - an useful biomarker in a combined predictive model for liver fibrosis staging in patients with chronic HCV infection. BMC Infectious Diseases 2014 14(Suppl 7):016.

Submit your next manuscript to BioMed Central and take full advantage of:

- Convenient online submission

- Thorough peer review

- No space constraints or color figure charges

- Immediate publication on acceptance

- Inclusion in PubMed, CAS, Scopus and Google Scholar

- Research which is freely available for redistribution

Submit your manuscript at www.biomedcentral.com/submit 